\title{
Super-thin inert chemical coating may help 'hide' stents from the body
}

A new stent applied with a nano-thin surface layer of an inert chemical compound has shown promise in opening and healing blocked coronary arteries without the life-threatening dangers of drug-eluting stents.

Stents have long been used to open up atherosclerotic arteries, where plaque has formed, in the heart, but often the vessels reclog in response to the body's reaction to the stent as a foreign object, resulting in in-stent scarring, known as restenosis. D rug-eluting stents were considered to represent an answer to restenosis, but the drug has been shown to interfere with the blood vessel's healing process, thereby increasing the risk of thrombosis and resulting in myocardial infarction. Although evidence suggests that thrombosis occurs in less than $1 \%$ of patients who receive drug-eluting stents, it is fatal in almost half of the cases.

The new stent has a surface application of Polyzene ${ }^{\circledR}-F$, a medically inert compound that has been used in humans for several years to coat tiny particles that embolize (block) arteries and treat a number of conditions, including malformed arteries. The Polyzene-F forms a coating that is 25,000 -times thinner than a human hair and is believed to 'hide' the stent from the body, thereby reducing restenosis and avoiding the thrombosisrelated problems associated with drug-eluting stents.

"O ur preliminary data show that the inside of the blood vessel with this polymercoated stent heals almost perfectly within 30 days, whereas with a drug-eluting stent, the blood vessel healing takes many months, if it ever happens," states Corrado Tamburino, principal investigator and professor of Cardiology at Ferrarotto $\mathrm{H}$ ospital (University of $\mathrm{C}$ atania, Italy). "O ur preliminary research suggests that this polymer-coated stent is a very promising solution to restenosis and thrombosis"

Results of the first use of this coated stent in humans are being presented at the 20th Annual International Symposium on Endovascular Therapy (FL, USA). In the Assessment of the Latest N on-Thrombogenic Angioplasty (ATLANTA) stent study at the University of Catania, 55 patients received the new surface treated stent, and 6 months follow-up information is availablefor 40 patients. At 6 months after implantation, the patients were studied using angiography and intravascular ultrasound. To date, the binary restenosis rate is low - less than $10 \%$ - and none of the stents have developed thrombosis.

An independent laboratory examined the Polyzene F-coated stents and also used optical coherence tomography (OCT) to image the vessels at the cellular level. OCT was performed at the time of the initial procedure and 6 months after implantation in 15 people $(27 \%)$ and results suggest that the Polyzene $F$ promotes healing, rather than prompting scarring.

With the introduction of drug-eluting stents 4 years ago, restenosis rates dropped to as low as $5-7 \%$ in some cases, according to studies. H owever, potentially deadly clots can form inside the drug-eluting stent, which researchers estimate kill 1000 or more people every year. To combat the thrombosis problem, patients who receive drug-eluting stents are often placed on dual antiplatelet therapy for at least 1 year.

"Although antiplatelet therapy delays the problem of thrombosis in drug-eluting stents, when the therapy is stopped, the problem returns," Tamburino claims. "However, continuing on antiplatelet therapy indefinitely is problematic, too, because it increases the risk of bleeding, and may prevent future surgeries."

The Polyzene-F-coated stents were designed to provide an answer to that problem. The antithrombotic, bacterialresistant coating ( $\sim 40 \mathrm{~nm}$ thick) essentially hides the stent so that the body does not recognize the device as a foreign object, as is the case for bare-metal stents. This, in turn, promotes the healing process so that the stent is incorporated into the body more naturally, preventing the formation of blood clots.

Source: 20th Annual International Symposium on Endovascular Therapy (ISET) www.ISET.org

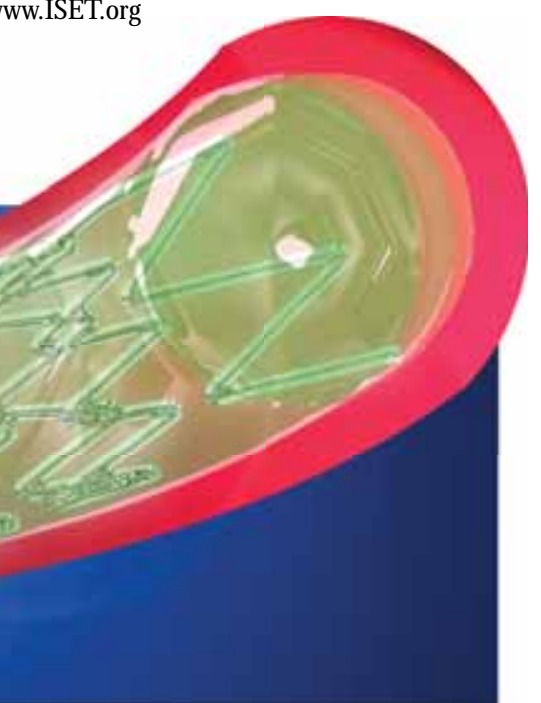




\section{Priority Paper Alerts}

Ventricular arrhythmias during clinical treadmill testing and prognosis. Dewey FE, Kapoor JR, Williams RS et al.: Arch. Intern. M ed. 168(2), 225-234 (2008). Aimed to evaluate the clinical correlates and prognostic significance of exercise-associated premature ventricular complexes (PVCs) during and after exercise testing. A total of 1847 heart failure-free patients who underwent clinical treadmill testing were studied. Logistic regression was used to evaluate the clinical and exercise test associations of exercise and recovery PVCs. Propensity score-adjusted COX survival analyses were used to evaluate the prognostic significance of exercise-associated PVCs. Of the 1847 subjects, 850 (46.0\%) developed exercise PVCs (median rate, $0.43 / \mathrm{min}$ ) and $620(33.6 \%)$ had recovery PVCs (median rate, $0.60 / \mathrm{min}$ ). During a 5.4-year mean follow-up, 161 subjects $(8.7 \%)$ died, and 53 of these deaths $(32.9 \%)$ were owing to cardiovascular causes. Recovery PVCs, but not exercise PVCs, were associated with 71-96\% greater propensity-adjusted mortality rates and occurrence of recovery PVCs reclassified 33.2\% of subjects with intermediate-risk Duke Treadmill Scores into higher-risk subgroups.

Causes of death in patients with acute myocardial infarction treated with angiotensin-converting enzyme inhibitors: findings from the Gruppo Italiano per lo Studio della Soprawivenza nell'Infarto (GISSI)-3 trial. Pedrazzini G, Santoro E, Latini R et al.: Am. Heart J. 155(2), 388-394 (2008). Aimed to describe the in-hospital causes of death in patients with acute myocardial infarction (MI) stratified to angiotensin converting enzyme (ACE) inhibitor treatment/no treatment, as described in the GISSI-3 trial. Patients receiving lisinopril had fewer in-hospital cardiac deaths than patients allocated to the no-lisinopril group (4.7 vs $5.3 \%, p=0.052$ ), corresponding to a $12 \%$ relative risk reduction. The risk of dying from cardiac rupture was reduced by $39 \%$ with lisinopril treatment. It is worth noting that the early beneficial effects of lisinopril observed at 6 weeks (eight fewer deaths per 1000 treated patients) were maintained for up to nearly 5 years (ten fewer deaths per 1000 treated patients). Thereby, researchers concluded that early administration of ACE inhibitors in unselected patients with acute MI should be considered standard therapy to reduce early deaths, specifically those due to cardiac rupture.

\section{COX-2 inhibitor may disrupt heart rhythm}

Celecoxib, the arthritis drug, induces heart arrhythmia in animal models independently of COX-2 inhibition, as demonstrated by study findings. Latest research indicated that the drug inhibits potassium ion channels and causes arrhythmia in D rosophila hearts and rat cardiac cells.

Adverse cardiovascular effects of selective COX-2 inhibition with drugs such as celecoxib and rofecoxib have caused much controversy. Previously, these effects have been attributed to reductions in antithrombotic, COX-2derived prostacyclin without a decrease in prothrombotic cOX-1-derived platelet thromboxane, tipping the balance towards a prothrombotic state.

"We now have shown an important new effect of [celecoxib] through a totally different pathway, one that is unrelated to the drug's effect as a pain reducer," states lead author Satpal Singh (State U niversity of Buffalo, NY, USA). "The adverse effect arising from this unexpected mechanism definitely needs to be studied more closely, because the potassium channels inhibited by the drug are present in heart, brain, and many other tissues in the human body."

Researchers first examined the effects of celecoxib in D rosophila, an important model for human cardiac pharmacology. The D rosophila heart rate was significantly reduced with exposure to low concentrations of celecoxib, and varied over time of drug exposure. At $1 \mu \mathrm{M}$, celecoxib induced intermittent arrhythmia in five of 17 cases, while $3 \mu \mathrm{M}$ celecoxib induced pronounced intermittent arrhythmia with several minutes of pronounced arrhythmic activity followed by several minutes of relatively regular heart beats.

'We now have shown an important new effect of [celecoxib] through a totally different pathway, one that is unrelated to

the drug's effect as a pain reducer.'

The effects of celecoxib on rat fetus cardiomyocytes, which establish a spontaneous beating rhythm in culture, was then examined. Celecoxib at $3 \mu \mathrm{M}$ significantly reduced the rate of beating in cardiomyocytes, and induced irregularity in the beat. The D rosophila gene and protein sequence databases were searched, but researchers found no $\mathrm{COX}$ like sequences, suggesting the effects of celecoxib were mediated by some other mechanism. Further analysis showed that celecoxib inhibited the delayed rectifier potassium current carried by Kv2 channels in Drosophila, and the rat analogues of these channels, Kv2.1 channels, were expressed in cultured human epithelial cells.

Research is ongoing, Singh explains "to try to determine whether the drug binds directly to the ion channels or to some other molecule, and if it acts by blocking the pore of the channel through which potassium ions travel or by some other mechanism."

Source: Frolov RV, Berim IG, Singh S: Inhibition of delayed rectifier potassium channels and induction of arrhythmia. A novel effect of celecoxib and the mechanism underlying it. J. Biol. Chem. 283, 1518-1524 (2008). 


\section{Intensive glucose-lowering arm of AC CORD trial halted}

The US National Heart, Lung and Blood Institute (N HLBI) has announced it is aborting the intensive glycemic control aspect of the Action to Control Cardiovascular Risk in Diabetes (ACCORD) trial in Type 2 diabetes patients, owing to an increased all-cause mortality among patients in the intensive compared with the standard gucose-lowering treatment arm.

"A thorough review of the data shows that the medical treatment strategy of intensively reducing blood sugar below current clinical guidelines causes harm in these especially high-risk patients with Type 2 diabetes," explained director Elizabeth $\mathrm{N}$ abel. "T hough we have stopped this part of the trial, we will continue to care for these participants, who now will receive the less-intensive standard treatment. In addition, we will continue to monitor the health of all participants, seek the underlying causes for this finding and carry on with other important research within ACCO RD."

A total of 10,251 patients with established Type 2 diabetes and who were at high risk of cardiovascular disease were enrolled in the ACCORD study, which was designed to compare three different approaches to reducing the high rate of cardiovascular events in these patients. The three approaches investigated were intensive versus standard glucose-lowering treatments, blood pressure and lipid-lowering treatments.

In the intensive glucose-lowering treatment arm, patients were treated with the aim of normalizing their glucose levels to a hemoglobin ( $\mathrm{Hb}$ b)Alc goal level of below $6 \%$. The standard treatment aimed to keep patients at a $\mathrm{HbAlc}$ level of $7-7.9 \%$, the average $\mathrm{HbAlc}$ level currently achieved in clinical practice in diabetics in the USA. Results demonstrated that the median $\mathrm{HbAlc}$ level achieved was 6.4 and $7.5 \%$ in the intensiveteatment and standard-treatment group, respectively.

After an average of 4 years treatment, 257 participants in the intensive-treatment group were found to have died compared with 203 in the standard-treatment group, translating into a 20\% higher rate of death in the intensive versus standard group. So far, the ACCORD researchers have not determined a specific cause for the increased deaths with intensive treatment and found no evidence that any medication or combination is responsible.

Source: N ational Heart, Lung, and Blood Institute ACCORD News Conference: 6 February 2008.

\section{Thrombus-aspiration percutaneous coronary intervention may offer better outcomes for myocardial infarction patients}

Patients with ST-segment myocardial infarction (STEMI) can achieve better reperfusion and clinical outcomes with thrombus-aspiration than with conventional percutaneous coronary intervention (PCI), latest research suggests.

With conventional $\mathrm{PCI}$, a balloon catheter is used before stent placement to reopen the occluded artery and re establish blood flow, but embolization of atherothrombotic debris can reduce myocardial reperfusion.

These latest findings come from a randomized trial carried out by Tone Svilas and colleagues from the University of Groningen ( $T$ he $N$ etherlands) to evaluate whether manual aspiration of the occluding thrombus with a catheter could improve myocardial reperfusion compared with conventional $\mathrm{PCl}$.
Prior to coronary angiography being performed, the researchers randomly assigned 1071 STEM I patients to undergo thrombus aspiration or conventional $\mathrm{PCl}$. Results demonstrated that thrombus aspiration was succesful in $72.9 \%$ of patients.

The primary end point was a myocardial blush grade of 0 or 1 , indicating absent or minimal myocardial reperfusion, respectively. Those patients who underwent thrombus aspiration showed significantly better myocardial reperfusion than those in the conventional $\mathrm{PCI}$ group, with 17.1 versus $26.3 \%$ of the patients having myocardial blush grade of 0 or 1 (risk ratio [RR]: 0.65; $p<0.001$ ).

Patients undergoing thrombusaspiration $\mathrm{PCl}$ were more likely to achieve complete resolution of ST -segment elevation compared with patients treated with conventional $\mathrm{PCl}$, at 56.6 versus $44.2 \%$ (RR: 1.28 ; $\mathrm{p}<0.001$ ).

Of all the participants, 5.2, 2.9 and $1.0 \%$ of patients with a myocardial blush grade of 0 or 1,2 and 3 , respectively, had died 30 days after $\mathrm{PCl}$ $(p=0.003)$. The rate of major cardiac adverse events was $14.1,8.8$ and $4.2 \%$, respectively $(p<0.001)$.

Svilas and coworkers conclude "T hrombus aspiration is applicable in a large majority of patients with STEM I, and it results in better reperfusion and clinical outcomes than conventional $\mathrm{PCl}$, irrespective of clinical and angiographic characteristics at baseline."

Source: Svilaas T, Vlaar PJ, van der Horst IC et al.: Thrombus aspiration during primary percutaneous Coronary intervention. N. Engl. J. M ed. 358, 557-567 (2008). 


\section{Possible 'clopidogrel rebound effect' identified in acute coronary syndrome patients after treatment cessation}

Acute coronary syndrome (ACS) patients who stop clopidogrel therapy may be at high risk for adverse events in the 90 days after treatment withdrawal, suggests a study published in the Journal of the American M edical Association.

John Rumsfeld (D enver Veterans Affairs M edical Center, CO, USA) and colleagues evaluated the incidence of adverse events in 3137 patients with ACS after they stopped treatment with clopidogrel. A total of 1568 medically treated patients were followed up for 196 days, and 1569 patients who underwent percutaneous coronary intervention (PCI) were followed up for 203 days.

The mean duration of clopidogrel treatment among all medically treated patients was 302 days. Among these patients, $17.1 \%$ experienced acute myocardial infarction (AM I) or death, with $60.8 \%$ of these events occurring in the first 90 days after clopidogrel cessation.
After adjusting for duration of clopidogrel treatment, the first 90-day interval after treatment cessation was found to be associated with a significantly higher risk for adverse events compared with the interval of 91-180 days (incidence rate ratio $[I R R]=1.98$ ).

$\mathrm{PCl}$ patients were treated with clopidogrel for a mean period of 278 days. D eath or AM I occurred in $7.9 \%$ of these patients, with $58.9 \%$ of events occurring 0-90 days after stopping clopidogrel treatment, $23.4 \%$ during $91-180$ days and $6.5 \%$ during $181-270$ days. Multivariable analysis revealed that the risk for adverse events was significantly increased during the first 90-day interval after stopping clopidogrel treatment (IRR: 1.82).

H owever, the researchers note that these findings "do not necessarily offset the benefits of clopidogrel therapy."
Rumsfeld and his colleagues conclude "We observed a clustering of adverse events in the initial 90 days after stopping clopidogrel among both medically-treated and $\mathrm{PCl}$-treated patients with ACS, supporting the possibility of a clopidogrel rebound effect."

They add: "Additional studies are needed to confirm the clustering of events after stopping clopidogrel, including associations with cardiovascular mortality and reasons for stopping clopidogrel, as well as to determine the mechanism of this phenomenon, and to identify strategies to reduce early events after clopidogrel cessation."

Source: Ho PM, Peterson ED, Wang $L$ et al.: Incidence of death and acute myocardial infarction associated with stopping clopidogrel after acute coronary syndrome. JAMA 299(5), 532-539 (2008).

\section{'N ewer' antihypertensive drugs not necessarily better than thiazide-type diuretics for metabolic syndrome patients}

Latest research from the Antihypertensive and Lipid-Lowering Treatment to Prevent $\mathrm{H}$ eart Attack Trial (ALLHAT) suggests that 'newer' antihypertensive drugs with more favorable metabolic profiles, such as calcium-channel blockers, $\alpha$-blockers or angiotensin-converting enzyme inhibitors, may not necessarily offer a better outcome compared with thiazide-type diuretics in hypertensive individuals with metabolic syndrome ( $M$ etS).

These findings originate from a subgroup analysis from ALLH AT, whereby outcomes in hypertensive individuals with and without $M$ etS treated with a thiazide-type diuretic (chlorthalidone), a calcium-channel blocker (amlodipine besylate), an $\alpha$-blocker (doxazosin mesylate) or an angiotensin-converting enzyme inhibitor (lisinopril).

$M$ etS was defined as hypertension plus at least two of the following: fasting serum glucose level of at least $100 \mathrm{mg} / \mathrm{dl}, \mathrm{BM}$ I of at least 30 , fasting triglyceride levels of at least $150 \mathrm{mg} / \mathrm{dl}$ and $\mathrm{HDL}$-cholesterol levels of less than $40 \mathrm{mg} / \mathrm{dl}$ in men or less than $50 \mathrm{mg} / \mathrm{dl}$ in women.

Significantly higher rates of heart failure were consistently found in $\mathrm{M}$ etS patients across all treatment comparisons. Higher rates for combined cardiovascular disease were observed with lisinopril-chlorthalidone (risk ratios [RRs]: 1.24 [1.09-1.40] and 1.10 [1.02-1.19], respectively) and doxazosin-chlorthalidone comparisons (RRs: 1.37 [1.19-1.58] and 1.18 [1.08-1.30], respectively) in black and nonblack participants with $\mathrm{M}$ etS.

In conclusion, the ALLH AT findings failed to support the preference for calcium-channel blockers, $\alpha$-blockers, or angiotensin-converting enzyme inhibitors compared with thiazide-type diuretics in patients with the $M$ etS, despite their more favorable metabolic profiles.

Source: Wright JT Jr, H arris-H ayward S, Pressel S et al.: Clinical outcomes by race in hypertensive patients with and without the metabolic syndrome: Antihypertensive and Lipid-Lowering Treatment to Prevent $\mathrm{H}$ eart Attack Trial (ALLHAT). Arch. Intern. Med. 168(2), 207-217 (2008). 


\section{N ovel protein kinase C inhibitor introduced as possible adjunct to percutaneous coronary intervention}

A novel compound that inhibits d-protein kinase $C$ activity could be used as an adjunctive therapy to limit ischemic and reperfusion injury during percutaneous coronary intervention $(\mathrm{PCI})$, according to preliminary research published in the journal Circulation.

"The goal of the treatment is to flood the heart damaged by the heart attack with the drug immediately before blood flow is restored and then again, immediately afterwards," explained lead investigator $M$ atthew Roe (Duke Clinical Research Institute, NC, USA). "We believe that bathing the area with this novel compound may block the damaging cascade of events that are triggered specifically by d-protein kinase $C$ when blood is restored to the heart muscle."

The investigational compound currently termed KAI-9803 - was tested in 154 patients with acute anterior ST-segment elevation myocardial infarction (STEMI) undergoing $\mathrm{PCl}$. Patients were then randomly assigned in a 2:1 manner to receive one of four doses of KAI-9803 $(0.05,0.5,1.25$ or $5.0 \mathrm{mg}$ ) or placebo, delivered in two doses via intracoronary injection before and after re-establishment of antegrade epicardial flow.

Results indicated that the rate of serious adverse events and other safety end points, including changes in QT

\section{Evidence suggests that the benefits of drug-eluting stents outweigh the risks}

After reviewing the benefits and risks associated with drug-eluting stents (DES) in current clinical data, US researchers have concluded that the weight of evidence suggests that the net clinical benefit of these stents outweighs the associated risks.

The authors stress in the Annals of Internal M edicine article that the apparent increased risk for late stent thrombosis with DES means that patients need to be screened for their ability to tolerate uninterrupted antiplatelet therapy "longer than is necessary for bare metal stents (BM S)".

Researchers examined patient-level data from major randomized trials comparing DES with BM S and found that late stent thrombosis (beyond 1 year) is significantly more frequent with both main types of DES than BMS. Yet, most studies, including meta-analyses of these trials, have not found any increase in death and myocardial infarction (M I), even after years of follow-up.

Weighing up the net clinical benefit, the authors claim that the $50-70 \%$ relative reduction in revascularization across most lesion subsets with DES versus BM $S$ has to be weighed against the potential absolute increase in late stent thrombosis of around $0.5 \%$. The number needed to treat with DES to prevent one restenosis ranges from between five and 20 , whereas the number needed to harm (one excess stent thrombosis event) is 200, the authors say, although they acknowledge that stent thrombosis is generally a more serious event than restenosis.

They conclude "Numerous studies have demonstrated that when used for 'on-label' indications, DES are effective at reducing restenosis and the need for repeat revascularization in all patients subgroups and lesion types, without an increase in late MI or excess mortality. However, there are few overall safety data from ade quately controlled trials on the use of DES for 'off-label' indications."

Source: Jeremias A, Kirtane A: Balancing efficacy and safety of drug-eluting stents in patients undergoing percutaneous coronary intervention. Ann. Intern. M ed. 148, 234-238 (2008). intervals and standard laboratory values after study drug administration, were similar among KAI-9803 and placebo groups. Furthermore, signs of potential drug activity were demonstrated with biomarker end points, indicating that further testing of KAI-9803 as an adjunctive therapy for STEM I is warranted.

Source: Direct Inhibition of Protein Kinase C Enzyme to Limit Total Infarct Size in Acute M yocardial Infarction (DELTA MI) investigators: intracoronary KAI-9803 as an adjunct to primary percutaneous coronary intervention for acute ST-segment elevation myocardial infarction. Circulation DOI: 10.1161/CIRCULATIONAH A.107.759167 (2008).
Possible renal risk for aprotinin plus ACE
inhibitors during
off-pump surgery

A UK team examined the association between aprotinin and renal dysfunction in 9012 patients undergoing cardiac surgery, 5434 of whom were operated on-pump and 3672 off-pump. The incidence of renal dysfunction in patients treated with aprotinin, tranexamic acid or no antifibrinolytic therapy in combination with or without preoperative angiotensin-converting enzyme (ACE) inhibitors was then analyzed.

Results indicated that only those patients undergoing off-pump surgery and treated with both ACE inhibitors and aprotinin were at high risk for postoperative renal dysfunction (odds ratio: $2.87 ; p=0.013$ ).

Source: M outon R, Finch D, D avies I, Binks A, Zachorowski K: Effect of aprotinin on renal dysfunction in patients undergoing on-pump and off-pump cardiac surgery: a retrospective observational study. Lancet 371, 475-482 (2008). 